\section{Orthodontic referral behaviour of West Sussex dentists}

\author{
0. A. Jackson, ${ }^{1}$ S. J. Cunningham, ${ }^{2}$ D. R. Moles $^{3}$ and J. R. Clark ${ }^{4}$
}

VERIFIABLE CPD PAPER
IN BRIEF

- Provides a snapshot of orthodontic referral decisions made by GDPs in West Sussex shortly after the introduction of the new NHS dental contract.

- Many dentists made appropriate referral decisions about malocclusions best managed in primary or secondary care.

- The majority of West Sussex dentists were not familiar with, or did not regularly use, the IOTN when making an orthodontic referral.

\begin{abstract}
Objectives 1) To examine the orthodontic referral behaviour of dentists and 2) to examine dentists' familiarity with the Index of Orthodontic Treatment Need (IOTN). Design Postal questionnaire survey. Setting West Sussex Primary Care Trust (PCT) from September to December 2006. Subjects and methods Questionnaires were sent to all dentists listed on the West Sussex PCT database, except those assumed not to make orthodontic referrals $(n=325)$. Results Two hundred and twenty-nine questionnaires were returned, representing a 70\% response rate. Fifty-two percent of dentists in West Sussex correctly identified which type of orthodontic provider to refer three different malocclusions using picture tests. Twenty percent of dentists made correct decisions on the timing of referral for three different malocclusions using picture tests. IOTN is not routinely used by $76 \%$ of West Sussex dentists when making an orthodontic referral. Conclusions This study provides evidence that there is a need for postgraduate training or the development of referral guidelines to assist West Sussex dentists in making referrals for orthodontic treatment to the most appropriate provider at the most appropriate time. If dentists are to act as gatekeepers of orthodontic provision on the NHS there is a need to provide more support and education for them about the use of IOTN.
\end{abstract}

\section{INTRODUCTION}

In the United Kingdom the majority of orthodontic treatment is carried out by specialist orthodontists (in hospital and practice settings), or by dentists with a special interest in orthodontics (DwSI), following referral from a general dental practitioner (GDP)., ${ }^{1,2}$ GDPs fulfil the important role of acting as gatekeepers for patients needing orthodontic treatment. In order to make best use of available orthodontic resources the referral should be made at the correct time to the most appropriate provider. Referrals made before the patient is ready to commence treatment may result

${ }^{1}$ Specialist Registrar in Orthodontics, ${ }^{4}$ Consultant Orthodontist, Orthodontic Department, St. Richard's Hospital, Spitalfields Lane, Chichester, West Sussex, P019 6SE; ${ }^{2}$ Senior Lecturer/Honorary Consultant and MSc/MOrth Programme Director, Orthodontic Unit, UCL Eastman Dental Institute, 256 Gray's Inn Road, London, WC1X 8LD; ${ }^{3}$ Professor of Oral Health Services Research, Director of Postgraduate Education and Research, Specialist in Dental Public Health, Peninsula Dental School, The John Bull Building, Research Way, Plymouth, PL6 8BU

${ }^{*}$ Correspondence to: Miss Orla Aine Jackson Email: orlajackson1@yahoo.co.uk

\section{Online article number E18}

Refereed Paper - accepted 22 June 2009

DOI: $10.1038 /$ sj.bdj.2009.979

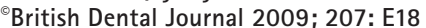

in unnecessary appointments, especially if the patient is then kept under review by the orthodontist. Equally, however, referrals made beyond the 'ideal' time may result in more complex and lengthy treatment being required. An initial referral to the most appropriate provider also avoids the need for an onward referral which may be wasteful of both orthodontic resources and patients' time. ${ }^{3}$

When the new dental contract was introduced in April 2006, NHS orthodontic treatment was restricted to patients with the most severe malocclusions, based on the Index of Orthodontic Treatment Need (IOTN). ${ }^{4}$ This index was originally developed for use by orthodontists ${ }^{5}$ and little is known about the familiarity of general dentists with its use. ${ }^{6}$

Recent changes in the organisation of dentistry in the $\mathrm{UK}^{7}$ have devolved funding and commissioning of dental services from a national to a local level and with this there has been an increased interest among primary care trusts (PCTs) in the referral process. This paper reports on a study carried out in West Sussex between September and December 2006 and funded by West Sussex Primary Care Trust.

\section{AIMS OF THE STUDY}

1. To determine whether dentists were able to refer patients to the most appropriate orthodontic provider

2. To determine whether dentists were able to make appropriate decisions regarding the timing of orthodontic referrals

3. To examine dentists' familiarity with the use of the Index of Orthodontic Treatment Need (IOTN).

\section{MATERIALS AND METHOD}

The study was a paper questionnairebased survey in West Sussex undertaken between September and December 2006. Questionnaires were sent to all dentists on the West Sussex PCT database except those assumed not to make orthodontic referrals, ie orthodontists, specialist dentists in prosthetics and oral surgery. The model for development of the questionnaire involved a thorough review of the literature, focus groups with consultant orthodontists and recently qualified dentists, along with faceto-face interviews with four local dentists. The information gained was examined for common themes about dentists' referral behaviours. The questionnaire was piloted 
on 20 local dentists requesting remarks about the readability and layout. Minor changes were made following this and the final questionnaire was then sent to 325 dentists. Reply paid envelopes were supplied and a request was made to return the questionnaire within two weeks of receipt. Second and third mailings were performed five and ten weeks later. Appeals were made to GDPs at the Local Dental Committee and British Dental Association meetings in an attempt to increase the response rate. A number of response enhancement strategies were used, including personalised participant information sheets and a statement ensuring that a summary of the survey's results would be sent to all dentists. Three separate mailings were undertaken to generate the best possible response rate.

The first section of the questionnaire identified demographic details. The subsequent sections used picture tests of a range of malocclusions, which had been agreed by the authors to show unequivocal features, to determine the appropriate timing of referral and appropriate orthodontic provider. The picture tests were in colour and supported by clinical details. In the three picture tests used to determine whether dentists referred to the most appropriate provider the questions were in two parts: firstly they were asked to state whether they considered the case would benefit from orthodontic treatment and, if so, which type of provider they would refer the patient to (Table 1). They were provided with the following options:

- Carry out the orthodontic treatment themselves

- Refer to a fellow general dental practitioner

- Refer to a specialist orthodontist in practice

- Refer to a hospital orthodontic consultant.

Additionally, three colour picture tests were used to determine perceptions of the correct timing of referral for particular malocclusions (Table 2). For each picture dentists were asked to state whether the case was either:

- At the right stage to make an orthodontic referral

- Too early to make an orthodontic referral

Table 1 The question asked dentists to decide whether a case would benefit from orthodontic treatment and, if so, which type of provider they would refer a patient to

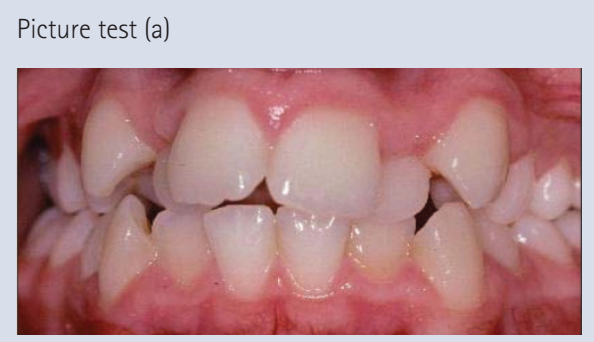

Clinical details:

Full permanent dentition

Crowding in the upper and lower labial segments Crossbite affecting right lateral incisor

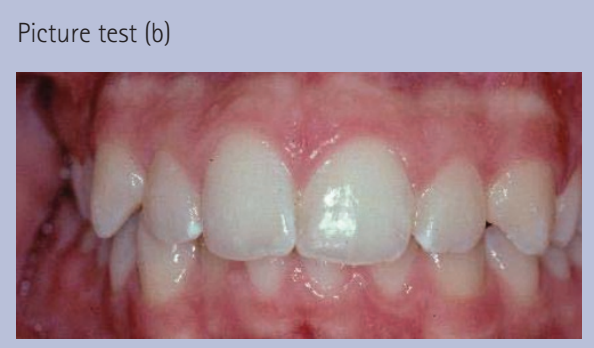

Clinical details:

Full permanent dentition

Mild crowding in the upper and lower labial segments

Slightly increased overjet ( $5 \mathrm{~mm}$ )

Picture test (c)

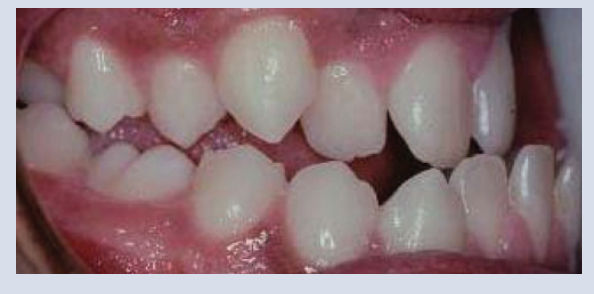

Clinical details:

Full permanent dentition

Moderate crowding in the upper and lower labia segments

Reverse overjet and large mandible

Table 2 The question used three picture tests to examine dentistsi opinions on the timing

of referral in three clinical scenarios

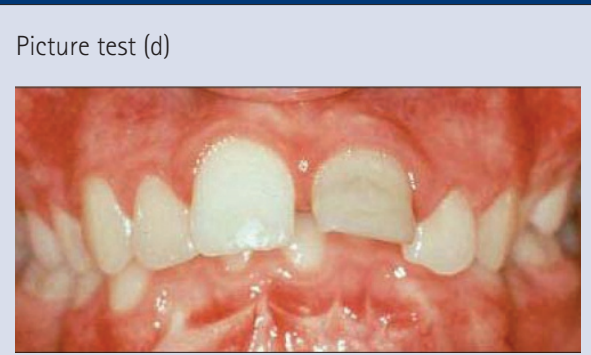

Clinical details:

Age 14

$10 \mathrm{~mm}$ overjet

Male patient

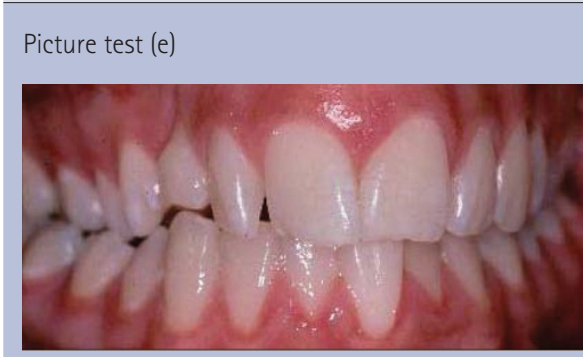

Clinical details:

Age 12

Upper left 3 erupted

Upper right $\mathrm{C}$ retained

Upper right 3 not palpable buccally

Picture test (f)

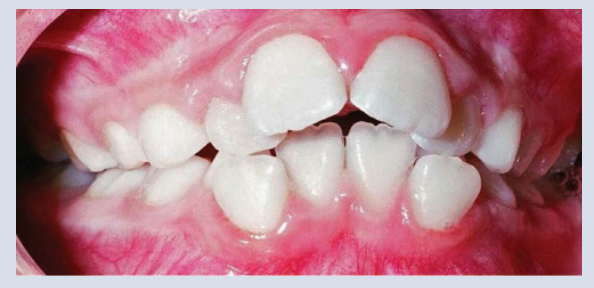

Clinical details:

Age 9

Mixed dentition

Normal overjet and overbite

No crossbites 
- Beyond the 'ideal' age for orthodontic referral.

\section{RESULTS}

\section{Demographic details}

The information obtained from the questionnaires was analysed using the computer statistical package SPSS V14 (SPSS Inc). Descriptive statistics were used together with cross tabulations and chi squared tests. Questionnaires were returned by 229 dentists and this represented a 70\% response rate. Sixty-two percent of respondents were male and 37\% were female, with $1 \%$ not indicating their gender. In addition, 23\% of respondents had a postgraduate qualification. The year and country of primary qualification are shown in Figures 1 and 2.

The profiles of responders and nonresponders were evaluated using the GDC online register to compare year of primary qualification, country of qualification and possession of a postgraduate qualification. No statistically significant responder bias was detected using these three factors.

\section{Picture tests}

Picture test (a) showed a patient in the full permanent dentition with crowding in the upper and lower labial segments and a crossbite affecting the right lateral incisor. The authors considered that this patient would benefit from orthodontic treatment and the treatment would ideally be provided by a specialist orthodontist in practice. All respondents agreed the case would benefit from treatment. Ninety percent (206) of respondents stated orthodontic treatment should be provided in a specialist orthodontic practice, $1(0.4 \%)$ dentist would have performed the treatment themselves, 5\% (10) would refer the case to a fellow dental practitioner, $3.5 \%$ (8) decided that this case should be referred to a hospital orthodontic consultant and 1\% of dentists (3) did not answer this section.

Picture test (b) showed a patient in the full permanent dentition with mild crowding in the upper and lower labial segments and a slightly increased overjet of $5 \mathrm{~mm}$. The authors considered that this was a very mild malocclusion and that the patient would not benefit significantly from orthodontic treatment. Of

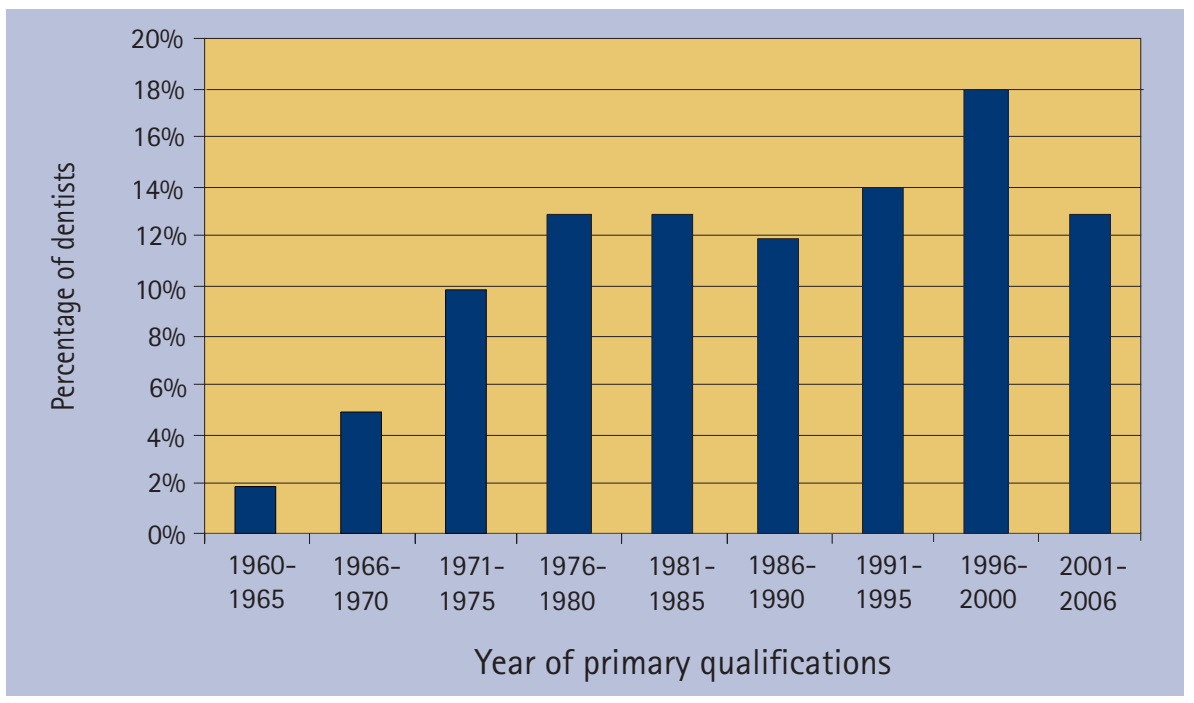

Fig. 1 Year of primary qualification of responders

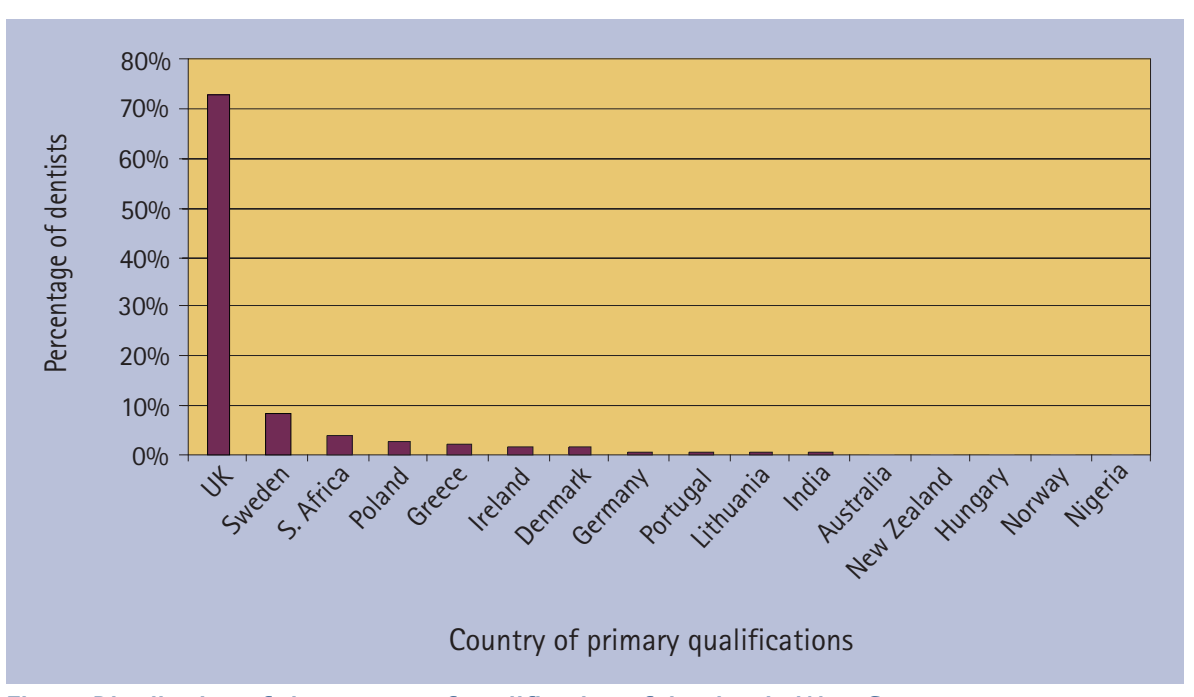

Fig. 2 Distribution of the country of qualification of dentists in West Sussex

the respondents, however, $25 \%$ of dentists stated that this case would benefit from orthodontic treatment. Of the 56 dentists who believed that the case warranted treatment, only 49 dentists (87.5\%) committed as to whom the patient should be referred and the referral was mainly to a practice-based specialist.

Picture test (c) showed a case in the full permanent dentition with moderate crowding in the upper and lower labial segments with a significant reverse overjet. The authors considered that this patient would benefit from combined orthodontic/ orthognathic surgical treatment and should be managed by a hospital orthodontic consultant. Of the respondents, 97\% agreed that the patient would benefit from orthodontic treatment, 25\% would refer this patient to a specialist practice and 73\% would refer to the hospital consultant. The remaining 2\% did not answer.
The total number of decisions made about where to refer patients for orthodontic treatment was 675, of which 543 (80\%) were considered appropriate by the authors. Whether dentists were 'correct' for all three decisions was also examined and $52 \%$ of dentists made the appropriate decision in all three cases. The ability to make the 'correct' decision in all cases was crosstabulated with two GDP characteristics (qualification within the last 10 years, ie since 1996, and possession of a postgraduate qualification) to determine if these had an influence on their referral behaviour. Qualification within the last 10 years was examined by the researchers because anecdotally it might be expected that dentists who have qualified more recently are more up-to-date with referral practices, while dentists with postgraduate qualifications might be expected to refer more appropriately. However, Tables 3 and 4 show that 
neither characteristic had a statistically significant effect on which provider these dentists chose to refer these cases to.

\section{Timing of referral}

Table 2 picture test (d) showed a 14-yearold male patient with a $10 \mathrm{~mm}$ overjet. The authors decided that this patient was beyond the 'ideal' age for an orthodontic referral because most orthodontists would have wished to use a functional appliance and such treatment is most successful when it coincides with the pubertal growth spurt. ${ }^{8}$ Eighty-three percent of responders (190) agreed with the view that the patient was beyond the age of an 'ideal' orthodontic referral, 16\% (37) believed the timing was just right and nearly $1 \%$ (1 dentist) stated that patient had been referred too early.

Picture test (e) showed a 12 year old patient with an erupted upper left canine and unerupted upper right canine with a retained deciduous canine and non-palpable upper right permanent canine. The authors' view was that this patient was beyond the 'ideal' age for an orthodontic referral as the lack of a buccal canine bulge on the right should have been noticed when the upper left canine began to erupt. The interceptive extraction of the deciduous canine at that stage might have improved the eruptive path of the upper right canine ${ }^{9}$ which is now likely to be impacted and require surgical exposure. Sixty-one percent (139) of the dentists in this study considered that the case was at the right stage for referral, 35\% (81) believed the case was beyond the 'ideal' age and 4\% (9) believed that it was too soon to make a referral.

Picture test (f) showed a patient aged 9 years in the mixed dentition with a normal overjet and overbite and no crossbites. The consensus view of the authors was that it was too early to make an orthodontic referral for this case as there were no obvious occlusal features which would have benefited from interceptive treatment. Seventy-five percent (170) of respondents agreed that it was too early to make an orthodontic referral for this case. However, $22 \%$ (50) thought that it was the right time to make the referral and 3\% (7) thought the case was at a stage beyond the 'ideal' for referral.

A total of 684 decisions were made about the timing of referrals and, of these,

Table 3 Cross-tabulation of appropriateness of the referral decision and the characteristic of 'Additional qualification'

\begin{tabular}{l|l|l|l|}
\multirow{2}{*}{ Decision type } & \multicolumn{3}{|c}{ Additional qualification } \\
\cline { 2 - 4 } & No & Yes & Total \\
\hline Appropriate decision & 91 dentists & 28 dentists & 119 dentists \\
\hline Inappropriate decision & 85 dentists & 25 dentists & 110 dentists \\
\hline Total & 176 dentists & 53 dentists & 229 dentists \\
\hline $\begin{array}{l}(p=1.00) \\
\text { Bu: there was no significant difference between those who made all the appropriate decisions and those who did not depending on whether } \\
\text { they held a postgraduate qualification or not. }\end{array}$
\end{tabular}

Table 4 Cross-tabulation of appropriateness of the referral decision and the characteristic of 'Qualified within the last ten years'

\begin{tabular}{|c|c|c|c|}
\hline \multirow[t]{2}{*}{ Decision type } & \multicolumn{3}{|c|}{ Qualified within the last 10 years } \\
\hline & No & Yes & Total \\
\hline Appropriate decision & 81 dentists & 38 dentists & 119 dentists \\
\hline Inappropriate decision & 78 dentists & 32 dentists & 110 dentists \\
\hline Total & 159 dentists & 70 dentists & 229 dentists \\
\hline
\end{tabular}

Table 5 Cross-tabulation of appropriateness of the referral decision and the characteristic of 'Additional qualification'

\begin{tabular}{l|l|l|l|}
\multirow{2}{*}{ Decision type } & \multicolumn{3}{|c}{ Additional qualification } \\
\cline { 2 - 4 } & No & Yes & Total \\
\hline Appropriate decision & 31 dentists & 14 dentists & 45 dentists \\
\hline Inappropriate decision & 145 dentists & 39 dentists & 183 dentists \\
\hline Total & 176 & 53 & 229 dentists \\
\hline $\begin{array}{l}(p=0.231) \\
\text { NB: there was no significant difference between those who made all the appropriate referral decisions and those who did not depending on } \\
\text { whether or not they held postgraduate qualification. }\end{array}$
\end{tabular}

441 (65\%) were considered to be appropriate in the consensus view of the authors. When the referral behaviour was examined further, however, it was calculated that only $20 \%$ (58) of dentists made the 'correct' decision about the timing of referral in all three cases. Cross-tabulation of the ability to make the appropriate decision with 'postgraduate qualification' and 'recent qualification' (within the previous 10 years), revealed that neither characteristic had a statistically significant effect on the timing of referral (Tables 5 and 6).

\section{Dentists' use of the Index of Orthodontic Treatment Need (IOTN)}

On April 12006 the IOTN was introduced into primary care dentistry in an attempt to provide a consistent reference for general dentists when referring patients for orthodontic treatment. Those patients with an IOTN score of greater than dental health component 3 and aesthetic component 6 are entitled to NHS treatment. Table 7 shows the responses dentists gave about their use of IOTN when making an orthodontic referral.

\section{DISCUSSION}

The response rate in a survey is usually an indicator of the quality of the data it provides. ${ }^{10}$ The response rate in this study was $70 \%$ and this is considered good, ${ }^{11}$ hence the results can be accepted as a reasonable representation of dentists' referral practices in West Sussex.

The process of making an effective referral for NHS orthodontic treatment involves:

- Determining the complexity of treating the malocclusion and deciding which orthodontic provider would be able 
Table 6 Cross-tabulation of appropriateness of the referral decision and the characteristic of 'Qualified within the last ten years'

\begin{tabular}{l|l|l|l|}
\multirow{2}{*}{ Decision type } & \multicolumn{3}{|c}{ Qualified within the last 10 years } \\
\cline { 2 - 4 } & No & Yes & Total \\
\hline Appropriate decision & 31 dentists & 14 dentists & 45 dentists \\
\hline Inappropriate decision & 128 dentists & 56 dentists & 184 dentists \\
\hline Total & 159 dentists & 70 dentists & 229 dentists \\
$\begin{array}{l}p=(1.00) \\
\text { NB: there was no significant difference between those who made all the appropriate referral decisions and those who did not depending on } \\
\text { whether or not they qualified within the last ten years. }\end{array}$
\end{tabular}

Table 7 Summary of dentistsi responses to the question: Do you use the Index

of Orthodontic Treatment Need (IOTN) when referring your patients?i

\begin{tabular}{|l|l|l}
\hline Response options & Number of responses & Percentage of dentists \\
\hline Never heard of IOTN & 12 & 5.2 \\
\hline Heard of IOTN but do not use it & 96 & 41.9 \\
\hline Occasionally use IOTN & 66 & 28.8 \\
\hline Often use IOTN & 39 & 17.0 \\
\hline Always use IOTN & 13 & 5.7 \\
\hline Total & $\mathbf{2 2 6}$ & $\mathbf{9 8 . 7}$ \\
\hline Missing responses & $\mathbf{3}$ & $\mathbf{1 . 3}$ \\
\hline Grand total & $\mathbf{2 2 9}$ & $\mathbf{1 0 0}$
\end{tabular}

to manage the case most effectively.

This requires local knowledge of the available orthodontic providers

- Assessing the stage of development of the dentition and whether the case is ready to commence orthodontic treatment

- Assessing the severity of the malocclusion and whether the case will be eligible to receive treatment, based on the IOTN score.

A decision about which provider to refer a patient to depends very much on the availability of the local orthodontic workforce. During the study period (September to December 2006) there were five hospital orthodontic consultants, 29 specialist orthodontic practitioners, one orthodontic senior dental officer and nine DwSI in orthodontics working in West Sussex. There was a reasonable geographic spread of these orthodontic providers within the county.

In this study the majority of dentists in West Sussex made appropriate decisions about which orthodontic provider to refer a moderately severe malocclusion requiring routine orthodontic care and a complex malocclusion requiring multidisciplinary care. Overall, however, only approximately half of dentists made what the authors considered to be the correct referral decision for all responses to the picture tests (a) to (c). A previous study found that a cohort of dentists in Birmingham made no distinction between hospital-based consultant services and other providers of orthodontic care. ${ }^{3}$ McComb et al. ${ }^{12}$ surveyed a group of dentists and reported that 51\% made all of their orthodontic referrals to the same orthodontic provider. The study by McComb and colleagues also attempted to discover what factors influenced the dentists' decisions to refer to a particular provider. The most important factors were reported to be length of treatment waiting list and the standard of treatment provided by the orthodontist. ${ }^{12}$ These studies reflect the fact that dentists have always been free to refer to the orthodontic provider of their choice. However, the decentralisation of funding for dentistry in 2006 has resulted in local commissioning and contracting for dental services and PCTs are now accountable for budget control and the efficient use of resources. It is inevitable that they will try to provide services by the most cost-effective means and to control the referral process more tightly.
The development of locally agreed referral guidelines, highlighting the roles of the different orthodontic providers, would provide dentists with sufficient information to make their referral to the most appropriate orthodontic provider.

The correct timing of an orthodontic referral ensures that the patient receives orthodontic treatment at the appropriate developmental stage. In a health service with a limited budget, referrals made too early could be construed as a waste of resources. The difficulty for the referring dentist, however, is diagnosing those cases which would benefit from interceptive orthodontic treatment and in whom a referral during the mixed dentition stage would be appropriate. Early referrals may be made by the dentist to circumvent a long waiting list and, while made with the patients' best interests in mind, may have the effect of lengthening the waiting list, thereby depriving other patients who may be ready to receive treatment. Conversely, when an orthodontic referral is received after the 'ideal' time there is a risk that treatment may be more complex or, indeed, impossible. In this study 65\% of the total number of decisions about the timing of referral made by West Sussex dentists agreed with the consensus view of the authors. However, only $20 \%$ of dentists made the correct decisions for all three of the picture tests (d) to (f). A previous study by Kisely et al. ${ }^{13}$ in Manchester assessed $26 \%$ of the referrals being made to an orthodontist as inappropriately timed. Such findings suggest there is a need for further education and development of referral guidelines.

Following the implementation of the new dental contract in April 2006, national restrictions were placed on access to NHS orthodontics for the first time. In order to limit orthodontic treatment to those cases which would derive the greatest dental health gain, the IOTN is now used to grade the severity of a malocclusion. Orthodontic providers have contracts which limit the treatment they provide to the most severe malocclusions based on IOTN, but little data exist to identify whether referring dentists are able to use the Index when making a referral.

In this study, 76\% of dentists did not routinely use the IOTN when making a referral. Current dental undergraduate curricula 
in the UK incorporate teaching on the IOTN but this has only been a recent addition to the curriculum. ${ }^{14}$ Dentists who qualified more than 10 years ago are unlikely to have had formal teaching or training in the use of the IOTN. In addition, dentists in West Sussex qualified in 16 different countries and it is possible that undergraduate curricula outside the UK do not incorporate occlusal indices such as the IOTN. If the IOTN is to be used successfully as a tool by referring dentists, considerable resources need to be directed at training. ${ }^{15}$ Burden et $a l .{ }^{15}$ reported on the trial of an IOTN learning package developed for use by dentists. The trial led to a reduction in the proportion of patients referred for treatment with mild malocclusions and helped dentists to identify cases where a borderline decision existed. ${ }^{15}$

The development of locally produced referral guidelines outlining when and where to make orthodontic referrals might help dentists make the best use of the available orthodontic manpower. This could be addressed by funding IOTN learning packages for individual practices or providing training courses. Alternatively, simple referral guidelines could be issued based on the essence of the IOTN to generate appropriate referrals, with the orthodontic providers being the final arbiters of the IOTN score.

\section{CONCLUSIONS}

- Approximately half of dentists (52\%) in West Sussex correctly identified which type of orthodontic provider to refer three different malocclusions using picture tests

- Only a minority (20\%) of dentists in West Sussex were able to correctly identify the appropriate timing of orthodontic referral for three different malocclusions using picture tests

- Neither the possession of a postgraduate qualification nor being recently qualified (within the past 10 years) influenced the ability to make the 'correct' decision with regard to orthodontic provider or timing of referral for orthodontic treatment

- 76\% of West Sussex dentists did not routinely use the Index of Orthodontic Treatment Need (IOTN) when making an orthodontic referral at the time of this survey (September-December 2006)

- Consideration should be given to whether the IOTN is an acceptable tool to be used by dentists when making a referral for orthodontic treatment, given the considerable knowledge gap in this area.

1. Guymon G, Buschang P H, Brown T J. Criteria used by general dentists to choose an orthodontist. J Clin Orthod 1999; 33: 87-93.
2. Stephens C D, Cook J. Attitudes of UK consultants to teledentistry as a means of providing orthodontic advice to dental practitioners and their patients. J Orthod 2002; 29: 137-142.

3. Langford J W, Ferguson J W. A comparison of consecutive orthodontics referrals seen by a consultant unit and specialist orthodontic practitioners. Br J Orthod 1995; 22: 347-352.

4. Department of Health. Strategic commissioning of primary care orthodontic services. London: Department of Health, 2006. http://www.dh.gov. uk/en/Publicationsandstatistics/Publications/ PublicationsPolicyAndGuidance/DH_4139176.

5. Brook P H, Shaw W C. The developmental of an index of orthodontic treatment priority. Eur J Orthod 1989 11: 309-320.

6. Richmond S, Shaw W C, O'Brien K D et al. The relationship between the index of orthodontic treatment need and consensus opinion of 74 dentists. Br Dent J 1995; 178: 370-374.

7. Department of Health. Primary care dental services: commissioning specialist dental services. London: Department of Heath, 2005. http://www.dh.gov. uk/en/Publicationsandstatistics/Publications/ PublicationsPolicyAndGuidance/DH_4123971.

8. Proffit W R, Fields H W, Sarver D M. Contemporary orthodontics. 4th ed. Philadelphia: Mosby Elsevier, 2007.

9. Ericson S, Kurol J. Early treatment of palatally erupting maxillary canines by extraction of the primary canines. Eur J Orthod 1988; 10: 283-295.

10. Oppenheim A N. Questionnaire design, interviewing and attitude measurement. London: Continuum International Publishing, 1992.

11. Tan R T, Burke F J T. Response rates to questionnaires mailed to dentists. A review of 77 publications. Int Dent J 1997; 47: 349-354.

12. McComb J, Wright J, O'Brien K. Dentist perceptions of orthodontic services. Br Dent J 1995: 178: 461-464.

13. Kisely S, Howell K, Green J. Pathways to orthodontic care. J Public Health Med 1997; 19: 148-155.

14. Derringer KA. Undergraduate orthodontic assessment and examination in UK dental schools. Br Dent J 2006; 201: 225-229.

15. Burden D J. An investigation of the association between overjet size, lip coverage, and traumatic injury to maxillary incisors. Eur J Orthod 1995; 17: 513-517. 